\title{
Characterization of a novel air-liquid interface biofilm of Pseudomonas fluorescens SBW25
}

Correspondence

Andrew J. Spiers

a.spiers@abertay.ac.uk

Received 14 October 2008

Revised 2 February 2009

Accepted 16 February 2009

\author{
Anna Koza, ${ }^{1}$ Paul D. Hallett, ${ }^{2}$ Christina D. Moon ${ }^{3}$ and Andrew J. Spiers ${ }^{1}$ \\ ${ }^{1}$ SIMBIOS Centre, Level 5 Kydd Building, University of Abertay Dundee, Bell Street, Dundee DD1 \\ $1 \mathrm{HG}, \mathrm{UK}$ \\ ${ }^{2}$ Scottish Crop Research Institute, Invergowrie, Dundee DD2 5DA, UK \\ ${ }^{3}$ AgResearch Limited, Grasslands Research Centre, Private Bag 11008, Palmerston North, New \\ Zealand
}

Pseudomonads are able to form a variety of biofilms that colonize the air-liquid (A-L) interface of static liquid microcosms, and differ in matrix composition, strength, resilience and degrees of attachment to the microcosm walls. From Pseudomonas fluorescens SBW25, mutants have evolved during prolonged adaptation-evolution experiments which produce robust biofilms of the physically cohesive class at the $A-L$ interface, and which have been well characterized. In this study we describe a novel A-L interface biofilm produced by SBW25 that is categorized as a viscous mass (VM)-class biofilm. Several metals were found to induce this biofilm in static King's B microcosms, including copper, iron, lead and manganese, and we have used iron to allow further examination of this structure. Iron was demonstrated to induce SBW25 to express cellulose, which provided the matrix of the biofilm, a weak structure that was readily destroyed by physical disturbance. This was confirmed in situ by a low (0.023-0.047 g) maximum deformation mass and relatively poor attachment as measured by crystal violet staining. Biofilm strength increased with increasing iron concentration, in contrast to attachment levels, which decreased with increasing iron. Furthermore, iron added to mature biofilms significantly increased strength, suggesting that iron also promotes interactions between cellulose fibres that increase matrix interconnectivity. Whilst weak attachment is important in maintaining the biofilm at the A-L interface, surface-interaction effects involving cellulose, which reduced surface tension by $\sim 3.8 \mathrm{mN} \mathrm{m}^{-1}$, may also contribute towards this localization. The fragility and viscoelastic nature of the biofilm were confirmed by controlled-stress amplitude sweep tests to characterize critical rheological parameters, which included a shear modulus of $0.75 \mathrm{~Pa}$, a zero shear viscosity of $0.24 \mathrm{~Pa} \mathrm{~s}^{-1}$ and a flow point of $0.028 \mathrm{~Pa}$. Growth and morphological data thus far support a non-specific metal-associated physiological, rather than mutational, origin for production of the SBW25 VM biofilm, which is an example of the versatility of bacteria to inhabit optimal niches within their environment.

\section{INTRODUCTION}

The formation of biofilms by bacteria is a key strategy in the colonization of natural environments, though biofilms are only one of a range of bacterial assemblages which occur. These range from isolated surface-attached bacteria, monolayers of associated bacteria forming microcolonies, and larger and more complex structures, including

Abbreviations: A-L, air-liquid; CBFS, Complementary Biofilm Forming Strain; EPS, extracellular polysaccharides; FM, floccular mass; MDM, maximum deformation mass; PC, physically cohesive; rdar, rough, dry and rugose; VM, viscous mass; WA, waxy aggregate; WS, Wrinkly Spreader.

Competing interests: The authors have declared that no competing interests exist. differentiated biofilms, to poorly attached or free-floating flocs and slime. Biofilm research has largely focused on submerged flow cell biofilms in which a surface-attached exopolysaccharide polymer matrix-based structure develops away from the surface into the flow of a nutrient and oxygen-rich growth medium, and where fluid flow and mass transfer affect biofilm development, structure and rheology (for recent reviews see Hall-Stoodley et al., 2004; Ramey et al., 2004; Branda et al., 2005; Battin et al., 2007; Danhorn \& Fuqua, 2007; Flemming et al., 2007, and references therein). In contrast, the partially saturated fluid-filled pores and networks found in soils, and the vascular systems and cavities found in plants, are significantly more complex environments for biofilmcolonizing bacteria and might be better approximated in 
the laboratory by low-flow-rate flow cells and static liquid microcosms with variable levels of oxygen.

We have been interested in the ability of bacteria to colonize the surface layer of static bodies of liquid, otherwise known as the air-liquid (A-L) interface (Gehrig, 2005; Spiers et al., 2002, 2006; Ude et al., 2006). In this favourable niche, nutrient and oxygen gradients are opposing, with access to abundant oxygen from the air above and nutrients in solution from below. The colonization of the $\mathrm{A}-\mathrm{L}$ interface requires a floating biofilm in which attachment at the meniscus to solid surfaces may be limited [A-L biofilms have been referred to as 'pellicles' (Branda et al., 2005)]. Both medical and environmental isolates of Escherichia and Salmonella displaying the rough, dry and rugose (rdar) phenotype are capable of producing cellulose matrix-based, wellattached and robust biofilms which colonize the A-L interface of static liquid microcosms (Römling, 2005). In a survey of plant-associated, soil- and river-isolated pseudomonads, $76 \%$ of isolates were able to form A-L biofilms after selection in static liquid microcosms, indicating that this is an evolutionarily deep-rooted ability with significant ecological advantages (Ude et al., 2006). These biofilms can be categorized on the basis of phenotype and physical robustness into physically cohesive (PC), floccular mass (FM), waxy aggregate (WA) and viscous mass (VM) classes (Spiers et al., 2006) (these are described in Table 1). Of these, the VM-class biofilms are the most fragile and appear not to rely on significant levels of meniscus attachment to maintain their position at the A-L interface. When gently disturbed, the biofilm mass slowly sinks like a dense viscous liquid. Bacterial assemblages such as VMclass biofilms, slimes and mucoidal colonies have received less attention than flow-cell biofilms, perhaps because of their fragile nature, but they are potentially important in high-humidity and aqueous environments including soil, the rhizosphere, and plant surfaces and tissues (Ramey et al., 2004; Danhorn \& Fuqua, 2007). The ability to form AL biofilms can confer significant fitness advantages, which have been demonstrated in vitro, whilst the cost to being a biofilm-forming mutant in an environment not suited to these structures is also measurable (Spiers et al., 2002; Gehrig, 2005; Spiers, 2007).

Mutants of Pseudomonas fluorescens SBW25 have been isolated that produce biofilms similar to those of the rdar mutants of Escherichia and Salmonella. In one particular SBW25 mutant known as the Wrinkly Spreader (WS), the single-base-pair mutation responsible for the overexpression of partially acetylated cellulose and the expression of an as-yet-unidentified fimbrae-like attachment factor necessary for biofilm formation have been identified (Spiers et al., 2002, 2003; Spiers \& Rainey, 2005; Bantinaki et al., 2007). In the WS, bacterial attachment at the meniscus region (the air-liquid-solid surface interface) leads to horizontal growth along the A-L interface (A-L biofilm meniscus growth may be similar in many respects to that seen in flow-cell systems where the biofilm is also directly attached to a solid surface). The physical strength of the biofilm resulting from interactions between cellulose fibre, attachment factor and lipopolysaccharide, plus the relative hydrophobicity of the structure, maintain it at the A-L interface (Spiers \& Rainey, 2005). Furthermore, when grown on agar plates, the WS forms a large, flat and wrinkled colony that is dramatically different from the smaller, smooth and rounded colony of wild-type SBW25. The Complementary Biofilm Forming Strain (CBFS) biofilm mutant was isolated from a cellulose-deficient SBW25 strain (Gehrig, 2005). This produces a thinner and more finely structured biofilm than the WS, and a distinctive colony morphology different from those of both the WS and wild-type SBW25. Although the underlying genetics of the CBFS are yet to be characterized

Table 1. Classes of $A-L$ interface biofilms produced by pseudomonads

Biofilm attributes were compiled from Spiers et al. (2006), Ude et al. (2006) and A. J. Spiers \& A. Koza, unpublished observations. Strength, ability to withstand weight applied to the top of the biofilm; Resilience, response to applied physical disturbance such as gentle or vigorous mixing; Attachment, connection to the microcosm vial walls in the meniscus region.

\begin{tabular}{|c|c|c|c|c|}
\hline \multirow[t]{2}{*}{ Attribute } & \multicolumn{4}{|c|}{ Biofilm class } \\
\hline & WA & FM & PC & VM \\
\hline Occurrence & Rare & Common & Common & Common \\
\hline Structure & $\begin{array}{l}\text { Single-piece structure, } \\
\text { rigid and brittle }\end{array}$ & Multiple elements or flocs & $\begin{array}{l}\text { Single-piece structure, flexible } \\
\text { and elastic }\end{array}$ & $\begin{array}{l}\text { One or more large, } \\
\text { viscous masses }\end{array}$ \\
\hline Strength & Strong & Medium & Strong & Weak \\
\hline Resilience & $\begin{array}{l}\text { Good, structure breaks } \\
\text { into small fragments }\end{array}$ & $\begin{array}{l}\text { Dissociates into flocs, but } \\
\text { flocs are hard to destroy }\end{array}$ & Very good & Very poor \\
\hline $\begin{array}{l}\text { Attachment and } \\
\text { meniscus growth }\end{array}$ & $\begin{array}{l}\text { Good attachment and } \\
\text { growth }\end{array}$ & $\begin{array}{l}\text { Some attachment and } \\
\text { growth }\end{array}$ & $\begin{array}{l}\text { Very good attachment and } \\
\text { growth }\end{array}$ & $\begin{array}{l}\text { Very poor attachment } \\
\text { and growth }\end{array}$ \\
\hline EPS & $\begin{array}{l}\text { No evidence of EPS, possible } \\
\text { cell-cell interactions }\end{array}$ & $\begin{array}{l}\text { Cellulose and evidence } \\
\text { for other EPS }\end{array}$ & $\begin{array}{l}\text { Cellulose and evidence for } \\
\text { other EPS }\end{array}$ & $\begin{array}{l}\text { Evidence for EPS but } \\
\text { not for cellulose }\end{array}$ \\
\hline
\end{tabular}


fully, the biofilm structure may be due to cell-cell interactions mediated by the expression of haemin storage system (Hms)-associated or regulated structures on the cell surface (Gehrig, 2005). Both the WS and CBFS form PCclass biofilms, as they produce physically cohesive, wellattached and strong biofilms at the $\mathrm{A}-\mathrm{L}$ interface [in addition to these two biofilms, SBW25 also produces a flow-cell cellulose-matrix-based biofilm (Villavicencio, 2000)].

We have discovered that SBW25 also produces a VM-class biofilm in static liquid microcosms in the presence of iron and various other metal ions. Here, we characterize the SBW25 VM biofilm in terms of induction, matrix component, strength and rheology, and attachment and localization to the A-L interface. Unlike the WS and CBFS biofilms formed by mutants derived from SBW25, this SBW25 VM biofilm appears to be a physiological response of the wild-type strain rather than the result of mutation.

\section{METHODS}

Bacteria, culture and microcosms. Bacterial strains are listed in Table 2 and were cultured in King's B (KB) medium (King et al., $1954)$ at $18-20{ }^{\circ} \mathrm{C}$ for all assays. $\mathrm{KB}$ plates contained $1.5 \%(\mathrm{w} / \mathrm{v})$ agar. Microcosms were $6 \mathrm{ml} \mathrm{KB}$ in $30 \mathrm{ml}$ universal glass vials inoculated with $60 \mu \mathrm{l}$ of an overnight shaken $\mathrm{KB}$ culture and were incubated statically with the lids loosely fixed to allow good aeration. Ferrous and ferric iron were provided by $\mathrm{FeSO}_{4}$ and $\mathrm{FeCl}_{3}$, respectively. Other metals were added in the form of $\mathrm{CuSO}_{4}, \mathrm{MnCl}_{3}, \mathrm{~Pb}\left(\mathrm{NO}_{3}\right)_{2}$ and $\mathrm{ZnCl}_{3}$. KB-Fe contained $1 \mu \mathrm{M} \mathrm{FeCl}_{3}$, and iron-chelated $\mathrm{KB}(\mathrm{KB}-\mathrm{DP} /$ $\mathrm{T})$ contained $20 \mu \mathrm{M}$ 2,2-dipyridyl (Sigma) and $0.1 \mu \mathrm{M}$ Tiron (1,2dihydroxybenzene-3,5-disulfonic acid; Fisher Chemicals).

Growth, colony morphology, twitching, bacterial density and hydrophobicity analyses. Bacterial growth and cell numbers were determined by $\mathrm{OD}_{600}$ measurements using a Spectronic Helios Epsilon spectrophotometer (Thermo Fisher Scientific) with $10 \mathrm{~mm}$ optical-path cuvettes. Colony morphology was examined on KB-DP/ $\mathrm{T}$ and $\mathrm{KB}-\mathrm{Fe}$ plates. Twitching was assessed by measuring the expansion of colonies across the agar-dish interface of $20 \mathrm{ml} \mathrm{KB-Fe}$ and $\mathrm{KB}-\mathrm{DP} / \mathrm{T}$ plates after $24 \mathrm{~h}$ incubation. The density of the compressed VM biofilm (i.e. cells and matrix but excluding liquid) was calculated from the weight of the pellet $(\mathrm{P})$ and volume of the supernatant $(S)$ after centrifugation of $6 \mathrm{ml} \mathrm{KB-Fe} \mathrm{microcosms} \mathrm{at}$ $16000 \mathrm{~g}$ for $1 \mathrm{~min}\left[\mathrm{P} /(6-\mathrm{S}) ; \mathrm{g} \mathrm{ml}^{-1}\right]$. The relative hydrophobicity $\left(H_{\mathrm{r}}\right)$ of SBW25 cells was determined in $\mathrm{KB}$ medium using a modification of the microbial adherence to hydrocarbons (MATH) assay (Spiers \& Rainey, 2005).
Tests for biofilm-forming mutants. Ten colonies were chosen randomly from $\mathrm{KB}$ plates spread with mature VM biofilm material from each of five independent KB-Fe microcosms incubated for $24 \mathrm{~h}$. Each of the 50 colonies was used to inoculate KB-Fe and KB-DP/T microcosms, which were inspected for growth and VM biofilm formation after 24. A $1 \mathrm{ml}$ sample of biofilm material was also recovered by centrifugation at $16000 \mathrm{~g}$ for $1 \mathrm{~min}$ from five independent $\mathrm{KB}-\mathrm{Fe}$ microcosms incubated for $24 \mathrm{~h}$. The supernatants were discarded and the pellets were washed in fresh $\mathrm{KB}$ to remove residual traces of iron before being resuspended in $1 \mathrm{ml} \mathrm{KB}$. Aliquots $(60 \mu \mathrm{l})$ were then used to inoculate $\mathrm{KB}-\mathrm{Fe}$ and $\mathrm{KB}-\mathrm{DP} / \mathrm{T}$ microcosms, which were inspected for growth and VM biofilm formation after $24 \mathrm{~h}$.

Cellulose detection and quantification. Qualitative assessments of cellulose expression were made by comparing the Congo red (CR) staining of colonies grown on plates containing $0.001 \%$ (w/v) CR (Sigma). Cellulose was also detected on plates containing $10 \mu \mathrm{g}$ Calcofluor $\mathrm{ml}^{-1}$ (Fluorescent Brightener 28; Sigma) and viewed using an AlphaImager HP System with a ML-26 UV transilluminator (GRI). A Leica DMR fluorescence microscope was used to view cellulose after staining with Calcofluor (Spiers et al., 2003). Cellulose levels were measured using a modified CR-binding assay (Spiers et al., 2003). Microcosms were gently mixed and CR was added to $0.001 \%(\mathrm{w} / \mathrm{v})$. After $2 \mathrm{~h}$, the samples were remixed and a $1 \mathrm{ml}$ aliquot was removed to determine the initial $A_{490}$. The samples were then centrifuged at $16000 \mathrm{~g}$ for $1 \mathrm{~min}$ to pellet the biofilm material, and the $A_{490}$ of the supernatant containing unbound CR was determined. CR binding was determined from the difference between $A_{490}$ measurements.

Biofilm strength, attachment and manipulation. Biofilm strength, as measured by the maximum deformation mass (MDM), was determined by carefully placing $0.0115 \mathrm{~g}$ glass balls onto the centre of biofilms until the biofilm broke or sank to the bottom of the microcosm (Spiers et al., 2003; Spiers \& Rainey, 2005). Attachment to the glass vials in the meniscus region was measured by crystal violet staining $\left(A_{570}\right)$, as described previously (Spiers et al., 2003). Both $\mathrm{MDM}$ and attachment were standardized for cell density using $\mathrm{OD}_{600}$ measurements. The involvement of attachment and buoyancy in the localization of the biofilm was assessed by slowly adding $\mathrm{KB}$ to mature VM biofilm microcosms $1 \mathrm{~cm}$ below the surface over $2 \mathrm{~h}$ and observing whether the biofilm moved upwards with the liquid level (which was adjusted by $5 \mathrm{~mm}$ ), flooded but remained in place or sank to the bottom of the microcosm vial. Aliquots $(60 \mu \mathrm{l})$ of water or $10 \mathrm{mM} \mathrm{FeCl}_{3}$ were similarly added to $\mathrm{VM}$ biofilm microcosms and allowed to equilibrate for $2 \mathrm{~h}$ before MDM assay to determine whether iron affected biofilm strength.

Biofilm rheology. Various parameters of the VM biofilm were measured by controlled-stress amplitude sweep tests using a rotational rheometer (MARS rheometer, Thermo Scientific fitted with $35 \mathrm{~mm}$ diameter parallel plates (PP35) and running HAAKE

Table 2. $P$. fluorescens SBW 25 and mutant strains used in this study

$\mathrm{Km}^{\mathrm{R}}$, kanamycin resistance.

\begin{tabular}{|c|c|c|}
\hline Strain & Description and genotype & Reference \\
\hline SBW25 & Wild-type P. fluorescens SBW25 & Rainey \& Bailey (1996) \\
\hline JB01 & Mutant overexpressing cellulose, SBW25 nptII-wss, $\mathrm{Km}^{\mathrm{R}}$ & Spiers et al. (2002) \\
\hline SM-13 & Mutant that does not express cellulose, SBW25 wssB:: mini-Tn5, $\mathrm{Km}^{\mathrm{R}}$ & Spiers et al. (2002) \\
\hline ViscA $^{-}$ & Mutant that does not express viscosin, SBW25 viscA::TnMod-OKm, $\mathrm{Km}^{\mathrm{R}}$ & de Bruijn et al. (2007) \\
\hline
\end{tabular}


RheoWin 3 software. Biofilm samples were from independent $\mathrm{KB}-\mathrm{Fe}$ microcosms incubated for $48 \mathrm{~h}$. Liquid media were removed from the microcosms by pipette before the biofilm was carefully poured onto the MARS plates and excess liquid drained off with a paper towel. Samples were placed onto the bottom plate and the gap between plates was closed to $0.2 \mathrm{~mm}$. An oscillating stress ramp was applied at an angular frequency of $0.5 \mathrm{~Hz}$ over a stress range of $0.001-1 \mathrm{~Pa}$ to cover the linear viscoelastic region and flow point. Measurements were made at $20 \pm 0.1{ }^{\circ} \mathrm{C}$, which was controlled with an integrated peltier thermal control unit. In an oscillating rheology test, the timedependent phase-shift between applied torque and angular displacement can be used to determine numerous rheological parameters (Mezger, 2006). The energy required for deformation is defined by the complex shear modulus $\left(G^{*}\right)$. In viscoelastic materials, $G^{*}$ comprises elastic (i.e. reversible) energy defined by the storage modulus $\left(G^{\prime}\right)$ and viscous (i.e. irreversible) energy defined by the loss modulus $\left(\mathrm{G}^{\prime \prime}\right)$. The ratio of viscous to elastic energy $\left(G^{\prime \prime}: G^{\prime}\right)$ defines the loss factor $(\tan \delta)$. This can be used to differentiate liquids $(\tan \delta>1)$ from gels or solids $(\tan \delta<1)$ (Mezger, 2006). The resistance to flow is defined as viscosity, with the zero-point viscosity $\left(\eta_{0}\right)$ recorded here. Zeropoint indicates that this was measured in the linear viscoelastic region, where $\eta, \mathrm{G}^{\prime}$ and $\mathrm{G}^{\prime \prime}$ do not change with the applied stress level. Above a critical stress level, all rheological parameters described above will decrease, resulting in the eventual breakdown of the material with the flow point occurring when $\mathrm{G}^{\prime}=\mathrm{G}^{\prime \prime}$.

Surface tension measurements. Surface tension was determined at $20{ }^{\circ} \mathrm{C}$ using $5 \mathrm{ml}$ samples of vortexed KB-Fe microcosms. Measurements were made with a Krüss K100 Mk2 tensiometer (Krüss) using an SV23 AI/PTFE conical sample vessel and platinum testing rod, following the surface and interfacial tension method with the Krüss Standard Rod and SFT Rod configuration template settings.

Statistical analysis. Statistical analysis was undertaken using JMP 7.0 (SAS Institute). Data were assumed to have normal distributions. Differences between means were tested by ANOVA, Tukey-Kramer honestly significant difference (HSD) and $t$ tests, which assumed unequal variances. Data are presented as mean $\pm \mathrm{SE}$, where appropriate.

\section{RESULTS}

\section{Preliminary description of the SBW25 VM biofilm}

Wild-type SBW25 growing in static KB microcosms containing $1 \mu \mathrm{M} \mathrm{FeCl}$ (KB-Fe) produced a VM-class biofilm within $24 \mathrm{~h}$, consisting of a large, viscous mass located at the A-L interface with little apparent attachment to the vial walls at the meniscus region (Fig. 1). In contrast, SBW25 cultures grown in iron-chelated KB (KB-DP/T) microcosms showed no sign of biofilm production after $24 \mathrm{~h}$, despite reaching a similar final population density as determined by $\mathrm{OD}_{600}$ measurements. Early tests indicated that no biofilm was produced in standard KB microcosms, confirming previous observations of SBW25 behaviour in comparison with WS biofilm formation (Spiers \& Rainey, 2005). However, subsequent tests with KB made with water from a different source suggested that low levels of iron contamination were sufficient to result in partial VM biofilm formation. Therefore, all subsequent experimentation compared the non-biofilm iron-chelated KB-DP/T control with the biofilm-inducing KB-Fe. When disturbed by gently shaking the vial, the VM biofilm sank slowly to the bottom, whilst vortexing destroyed all structure and left no visible clumps of material remaining. The relative weakness of the VM biofilm was confirmed by maximum deformation mass measurements, whereby only 0.023 $0.047 \mathrm{~g}$ of glass balls was able to be supported by the biofilm before breaking, in contrast to the more robust PCclass WS biofilm, which can support $\sim 0.36 \mathrm{~g}$ (Spiers \& Rainey, 2005). Extracellular cellulose fibres were observed by fluorescence microscopy in VM biofilm samples stained with Calcofluor, suggesting that cellulose provided the main structural (matrix) element of this weak biofilm (Fig. 2). In addition, colonies recovered from the VM biofilm showed no significant difference in morphology from those of standard KB microcosms. This is in contrast to the biofilm-forming mutants WS and CBFS, which produce colonies that are morphologically distinct from those of the wild-type SBW25, suggesting that the VM biofilm results from physiological induction by iron rather than from mutation.

\section{Induction of the SBW25 VM biofilm}

We examined the effect of $\mathrm{FeCl}_{3}$ concentration on the induction and development of the SBW25 VM biofilm via measurements of growth, biofilm strength and attachment. As expected, SBW25 growth was found to increase with the addition of iron in the range of $0-50 \mu \mathrm{M} \mathrm{FeCl}_{3}$ (Fig. 3a); thus, measurements of biofilm strength and attachment were standardized for cell density differences. Biofilm strength, as determined by the MDM assay, was found to increase with increasing $\mathrm{FeCl}_{3}$ concentration (Fig. 3b), demonstrating a positive response to the presence of iron; a $1.85 \times$ increase in MDM was found between biofilms

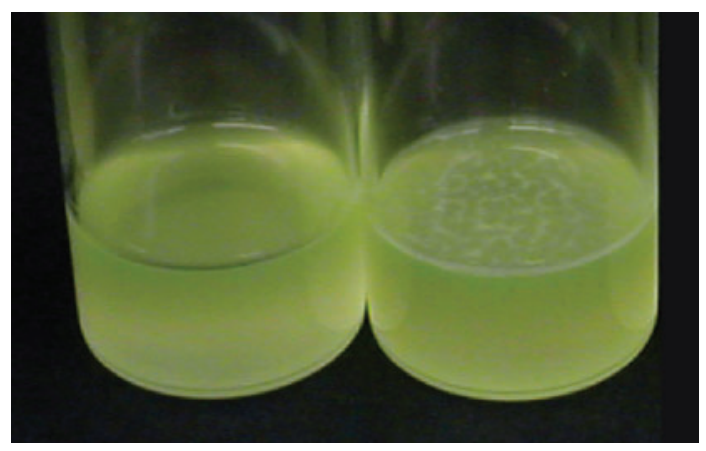

Fig. 1. A novel VM-class biofilm is induced in $P$. fluorescens SBW25 by iron when incubated in static KB microcosms. SBW25 cultures in $6 \mathrm{ml} \mathrm{KB}$ liquid microcosms show signs of growth, including increased turbidity and siderophore expression near the top of the liquid column, within $24 \mathrm{~h}$ at $18-22{ }^{\circ} \mathrm{C}$. Shown on the left is a KB-DP/T microcosm in which all free iron is chelated; on the right is a $\mathrm{KB}-\mathrm{Fe}$ microcosm with $1 \mu \mathrm{M} \mathrm{FeCl}_{3}$ added, in which a VM biofilm has developed at the $A-L$ interface. This has the appearance of several globular, shiny masses which do not pierce the surface or project into the air. 
(a)
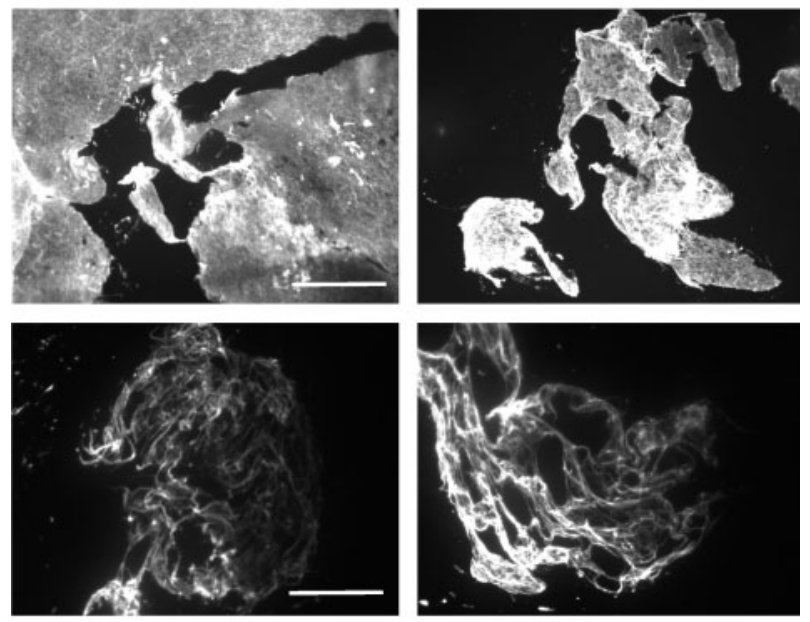

(b) SBW25 SM-13 JB01
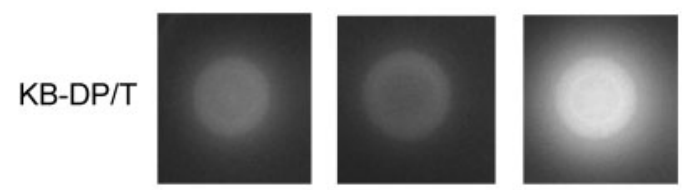

KB-Fe
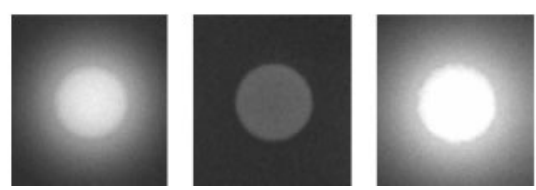

Fig. 2. Iron induces the expression of cellulose by $P$. fluorescens SBW25. Biofilm samples and colonies stained with Calcofluor fluoresce strongly when cellulose is present. (a) Fluorescence microscopy images of VM biofilm material from KB-Fe microcosms showing large aggregates of cellulose-containing material at $\times 10$ magnification (top images) and cellulose fibres at $\times 40$ magnification (bottom images). Bars: upper panels, $250 \mu \mathrm{m}$; lower panels, $60 \mu \mathrm{m}$. (b) Images of SBW25, SM-13 and JB01 colonies grown on KB-DP/T and KB-Fe plates containing Calcofluor (SM-13 is a cellulose-deficient mutant, whilst JB01 constitutively expresses cellulose). VM biofilms and colonies were imaged after $24 \mathrm{~h}$.

grown with 0.1 and those grown with $50 \mu \mathrm{M} \mathrm{FeCl}_{3}$ ( $t$ test, $\left.\mathrm{DF}_{13.42}, t_{5.853}, P<0.0001\right)$. In contrast, biofilm attachment levels were found to decrease with increasing levels of iron (Fig. 3c), and a $0.75 \times$ decrease in attachment was observed between 0.1 and $50 \mu \mathrm{M} \mathrm{FeCl}$ ( $t$ test, $\mathrm{DF}_{12.52}, t_{-2.075}$, $P=0.0592$ ). SBW25 responded similarly to ferrous iron (in the form of $\mathrm{FeSO}_{4}$ ) and produced a VM biofilm. Iron is known to depress twitching behaviour in Pseudomonas aeruginosa PAO1 and to subsequently enhance biofilm development in flow-cell systems (Singh et al., 2002). In contrast, SBW25 twitching motility, as assessed by agar plate measurements, increased up to 2.3-fold on KB plates with $1-10 \mu \mathrm{M} \mathrm{FeCl}$ compared with $\mathrm{KB}-\mathrm{DP} / \mathrm{T}$ plates
(ANOVA, $\mathrm{DF}_{2.21}, F_{18.45}, P<0.0001$ ), though this assay did not account for growth differences. This increased SBW25 twitching might account for the decrease in levels of VM biofilm attachment in response to iron, as in PAO1, twitching cells do not form cell clusters or go on to develop biofilms.

A mutant (PBR840) deficient in the production of the iron-scavenging siderophore pyoverdine (PVD), a central component in SBW25 iron homeostasis (Moon et al., 2008), was also found to be able to form VM biofilms with either $\mathrm{FeSO}_{4}$ or $\mathrm{FeCl}_{3}$, suggesting that the induction mechanism is independent of this iron-uptake pathway. We further tested the specificity of iron in biofilm induction, and found that several other compounds were able to induce the VM biofilm, including $5 \mu \mathrm{M} \mathrm{CuSO}$, $25 \mu \mathrm{M} \mathrm{MnCl}_{3}$ and $25 \mu \mathrm{M} \mathrm{Pb}\left(\mathrm{NO}_{3}\right)_{2}$, indicating that induction is not iron-specific and may be a common response to a wider range of metals. However, of the metals tested, induction was not seen with $0.1-50 \mu \mathrm{M} \mathrm{ZnCl}_{3}$, and in addition, the $\mathrm{MgCl}_{2}$ in $\mathrm{KB}$ was found not to be necessary for induction in the presence of iron.

We examined SBW25 VM biofilm cells and derived cultures for the presence of iron-sensitive biofilm-forming mutants, which might provide an alternative explanation for the development of VM biofilms. We tested 50 randomly selected and independently isolated colonies from VM biofilms and found that none produced biofilms in KB-DP/T microcosms, but all produced biofilms in KBFe microcosms. Similarly, VM biofilm inocula only produced VM biofilms in $\mathrm{KB}-\mathrm{Fe}$ microcosms and not in $\mathrm{KB}-\mathrm{DP} / \mathrm{T}$ microcosms. If mutation was responsible for VM biofilm formation in $\mathrm{KB}-\mathrm{Fe}$ microcosms, then we would have expected to be able to isolate mutants that formed VM biofilms independently of iron in subsequent KB-Fe microcosms. These observations support our conclusion that VM biofilm formation is most probably a non-specific physiological response by SBW25 to iron in static KB microcosms. Nonetheless, iron can be used to induce this biofilm and we have used it as a representative metal to allow further investigation of the VM biofilm.

\section{Cellulose has a structural role as the matrix element of the VM biofilm}

Cellulose expression by SBW25 has previously been demonstrated by staining samples with Calcofluor and observation by fluorescence microscopy (Spiers et al., 2003; Spiers \& Rainey, 2005). Using the same technique, we identified the presence of large masses of extracellular cellulose fibre in VM biofilm samples (Fig. 2a). We were able to detect low levels of cellulose expression by SBW25 grown in $\mathrm{KB}$, confirming earlier observations of unregulated, basal expression (Spiers et al., 2003), but were not able to detect cellulose in SBW25 samples grown in KB$\mathrm{DP} / \mathrm{T}$. Cellulose levels, as measured by the CR-binding assay, were 1.2 times greater in $\mathrm{KB}-\mathrm{Fe}$ microcosms containing VM biofilms than in $\mathrm{KB}-\mathrm{DP} / \mathrm{T}$ microcosms 

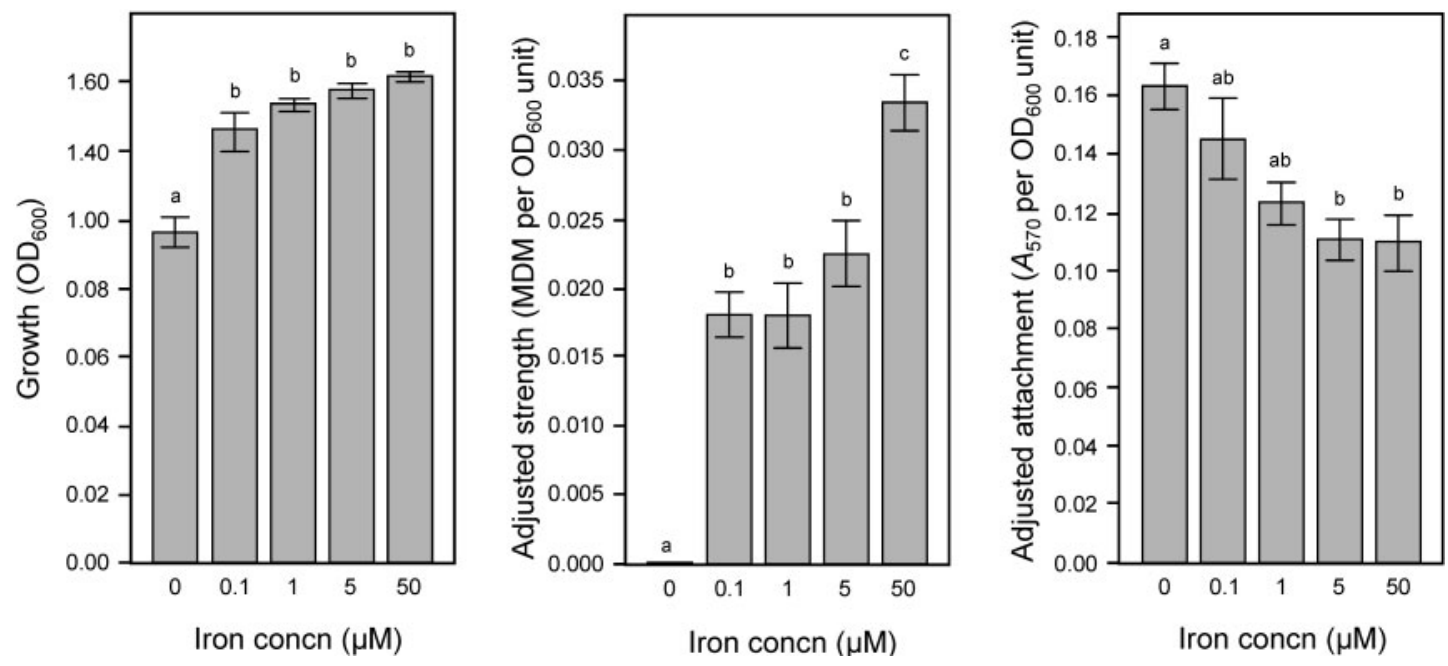

Fig. 3. VM biofilm development responds to increasing levels of $\mathrm{FeCl}_{3}$. Biofilm development was assessed by parallel measurements of growth, strength and attachment. (a) Growth determined by $\mathrm{OD}_{600}$ measurements. (b) Biofilm strength as determined by MDM assay and adjusted for growth (grams per $\mathrm{OD}_{600}$ unit). (c) Biofilm attachment as determined by crystal violet staining and adjusted for growth $\left(A_{570}\right.$ units per $\mathrm{OD}_{600}$ unit). Note that $0 \mu \mathrm{M}$ iron refers to KB-DP/T, whilst $0.1-50 \mu \mathrm{M}$ iron indicates $\mathrm{KB}$ with that amount of $\mathrm{FeCl}_{3}$ added to the medium. SBW25 VM biofilms were assayed after $24 \mathrm{~h}$ incubation. MDM measurements were made first, and the contents of the microcosms tipped into a $15 \mathrm{ml}$ tube and vortexed to break up the biofilm material to allow growth measurements. The emptied microcosm vials were stained to determine attachment levels. Means were compared by Tukey-Kramer $\operatorname{HSD}\left(\mathrm{q}^{*}{ }_{2.875}, \alpha_{0.05}\right)$; samples not connected by the same letter are significantly different. Means \pm SEM are shown $(n=8)$.

which did not contain biofilms ( $t$ test, $\mathrm{DF}_{11.43}, t_{10.81}$, $P<0.0001)$. Finally, we also examined cellulose expression in colonies grown on plates containing Calcofluor (Fig. $2 \mathrm{~b}$ ), which again demonstrated that iron induced SBW25 to express cellulose.

Although iron clearly induced the expression of cellulose by SBW25 in the VM biofilm, it was not clear whether cellulose was necessary for VM biofilm formation (in the sense that cellulose provided the biofilm matrix and was largely responsible for the biofilm structure). In order to determine this, we investigated VM biofilm formation using two genetically defined SBW25 mutants. We first determined that the cellulose-deficient strain SM-13 was unable to form a VM biofilm in static KB-Fe microcosms. $\mathrm{OD}_{600}$ measurements confirmed that SM-13 population sizes were similar to those of SBW25 in $\mathrm{KB}-\mathrm{Fe}$, indicating that reduced cell numbers could not explain the lack of biofilm formation. Therefore, cellulose production is essential for VM biofilm formation. These findings are further confirmed by an examination of JB01, a strain which constitutively expresses cellulose, and has previously been reported to produce a very weak non-WS-like biofilm (Spiers et al., 2002). In KB-DP/T, JB01 produced a biofilm visually indistinguishable from and of similar strength to the SBW25 VM biofilm in KB-Fe, but the JB01 biofilm in $\mathrm{KB}-\mathrm{Fe}$ was 4.5 -fold stronger than the VM biofilm in KB-Fe (Tukey-Kramer HSD, $\mathrm{q}_{2.521}^{*}, \alpha, 0.05$ ), suggesting either that JB01 expressed more cellulose in KB-Fe than in KB-DP/T, or that the presence of extracellular iron resulted in a stronger biofilm by promoting extra matrix connectivity. The former is supported by a comparison of Calcofluorstained colonies, which suggests that JB01 grown on KB-Fe plates expresses more cellulose than either JB01 on KB-DP/ T or SBW25 on KB-Fe, which express similar amounts of cellulose (Fig. 2b), whilst the latter is addressed below.

\section{Iron promotes matrix connectivity in the VM biofilm}

We examined whether iron can promote interactions between cellulose fibres to increase matrix connectivity. SBW25 is known to express small amounts of cellulose in $\mathrm{KB}$ (Spiers et al., 2003), and it is possible that this basal level of cellulose is cross-linked by iron to produce the VM biofilm without requiring further cellulose expression, which iron has been shown to induce (notwithstanding the fact that cellulose expression was undetectable in KB-DP/T as determined by Calcofluor fluorescence microscopy). We tested this possibility by adding $100 \mu \mathrm{M} \mathrm{FeCl}_{3}$ to nonbiofilm-forming cultures of SBW25 and SM-13 KB-DP/T microcosms, and in neither case did the cultures condense to form a biofilm or gel-like mass at the bottom of the vial within $2 \mathrm{~h}$. However, the addition of $100 \mu \mathrm{M} \mathrm{FeCl}_{3}$ to mature VM biofilms significantly increased biofilm strength after $2 \mathrm{~h}$ by 1.7 -fold, compared with VM biofilms to which water-only additions were made ( $t$ test, $\mathrm{DF}_{12.99}$, $\left.t_{-2.078}, P=0.0580\right)$. These results support the hypothesis 
that iron can promote extra matrix connectivity within existing biofilms, and results in stronger biofilms.

\section{Rheometry of the VM biofilm}

Increasingly, flow-cell biofilms have been directly examined by rheological measurements in order to define their rheological (structural) characteristics [for a brief introduction and a review of rheology, see Janmey \& Schliwa (2008) and Janmey et al. (2007), respectively]. As these biofilms develop in a steady flow of nutrients, of key interest has been how the biofilm structure resists the pressure of the liquid moving across the surface of the biofilm (the pressure acting on one surface is known as 'shear'). Flow-cell biofilms appear to have the general characteristics of a viscoelastic structure (Hall-Stoodley et al., 2004) which is able to absorb the energy of low levels of shear by slight deformation without catastrophic failure (i.e. breaking into pieces), and release the stored energy by recovery when the shear is reduced. Above a critical shear (known as the flow point), the structure begins to flow like a viscous fluid. Below this point, rheological behaviour is constant in what is known as the linear viscoelastic region. In this region, a structure can be characterized by several parameters, such as the storage $\left(G^{\prime}\right)$, loss $\left(G^{\prime \prime}\right)$ and shear $\left(G^{*}\right)$ moduli, loss factor $(\tan \delta)$, flow point and zero shear viscosity $\left(\eta_{0}\right)$ (see Methods for further information), which can be used to determine whether a structure has a viscoelastic nature.

We determined that the VM biofilm was also a viscoelastic solid, with $\tan \delta<1$ at $0.47 \pm 0.03$. As expected with such structures, the elastic portion of viscoelasticity $\left(\mathrm{G}^{\prime}\right)$ decreased with increasing shear stress, indicating shear-thinning behaviour, and liquid flow occurred at $0.028 \pm 0.004 \mathrm{~Pa}$. The VM biofilm was found to have a $\mathrm{G}^{*}$ of $0.75 \pm 0.16 \mathrm{~Pa}$ and $\eta_{0}$ of $0.24 \pm 0.05 \mathrm{~Pa} \mathrm{~s}^{-1}$, which are indicators of structural stability and resistance to deformation.

\section{Localization of the VM biofilm to the A-L interface}

The low levels of attachment of the SBW25 VM biofilm to the vial walls and the ready sinking after gentle disturbance raise the question of how the SBW25 VM biofilm is localized to the A-L interface of the $\mathrm{KB}$ microcosm. Here we considered four possibilities: buoyancy (where the mass of the biofilm is less than that of the displaced $\mathrm{KB}$ ), bacterial cell hydrophobicity (resulting in the cells having a strong preference to be at the surface rather than being submerged), weak meniscus attachment (as has already been observed, Fig. 3c) and surface interactions (where other interactions of biofilm components with the surface contribute to the localization of the biofilm). The SBW25 VM biofilm was more dense than $\mathrm{KB}$ medium $\left(0.47 \pm 0.09 \mathrm{~g} \mathrm{ml}^{-1}\right.$ compared with $\left.0.303 \mathrm{~g} \mathrm{ml}^{-1}\right)$ and no significant difference in relative hydrophobicity was found between biofilm samples from $\mathrm{KB}-\mathrm{Fe}$ microcosms and
SBW25 cultures grown in KB-DP/T ( $t$ test, $\mathrm{DF}_{5.182}, t_{-0.7164}$, $P=0.5047$ ), indicating that buoyancy and hydrophobicity do not contribute to the localization of the SBW25 VM biofilm to the A-L interface. In contrast, we could demonstrate that the weak attachment was important by increasing the liquid level by $5 \mathrm{~mm}$ in mature SBW25 VM biofilm microcosms. Rather than being moved upwards, the biofilms were flooded by a layer of $\mathrm{KB}$, indicating that they were maintained in place by attachment to the vial walls. However, the flooded biofilms clearly sagged in the middle (Fig. 4), suggesting that some form of surface interaction was also involved in the localization of the biofilm to the A-L interface.

Possible surface interactions might be revealed by altered A-L surface tension $(\gamma)$, as $\gamma$ is altered by adsorption layers formed by surface-active agents such as amphipathic compounds and surfactants (Van Hamme et al., 2006). SBW25 is known to produce the surfactant viscosin (de Bruijn et al., 2007), which is not required for VM biofilm formation, as the viscosin-deficient mutant $\mathrm{ViscA}^{-}$was found to produce an SBW25 VM-like biofilm in KB-Fe (data not shown). We determined the $\gamma$ of vortexed SBW25 VM biofilms and VM-like biofilms from ViscA ${ }^{-}$, and of static cultures of SM-13 in KB-Fe microcosms (Fig. 5) (although all measurements were made using $\mathrm{KB}-\mathrm{Fe}$, we noted that $\gamma$ of sterile KB-Fe was 1.06-fold that of KB-DP/T ( $t$ test, $\left.\mathrm{DF}_{5.621}, t_{9.268}, P=0.0001\right)$. These data indicate that a significant reduction of $\gamma\left(\sim 19.5 \mathrm{mN} \mathrm{m}^{-1}\right)$ could be attributed to viscosin, as expected, and a smaller reduction $\left(\sim 3.8 \mathrm{mN} \mathrm{m}^{-1}\right)$ to cellulose, demonstrating that cellulose can act as a surface-active agent, and might enable an interaction between the A-L interface and the SBW25 VM

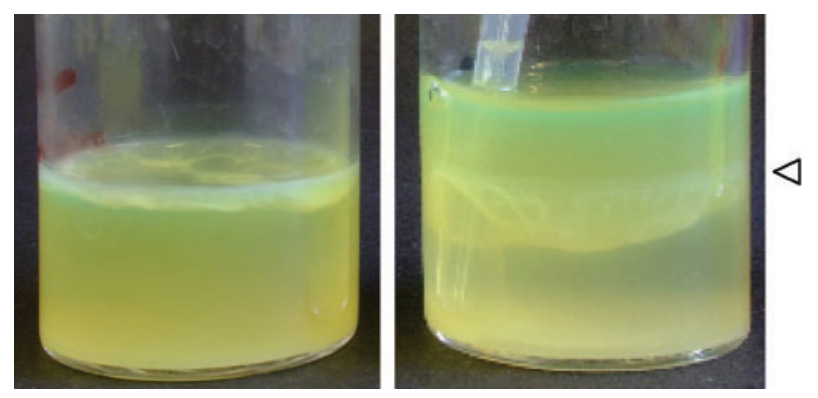

Fig. 4. Sagging of flooded SBW25 VM biofilms suggests that surface interactions help maintain the biofilm at the $A-L$ interface. Shown are two mature SBW25 VM biofilms in KB-Fe microcosms; the one on the right has had extra KB medium added below the surface of the biofilm over a period of $2 \mathrm{~h}$ to raise the liquid level by $5 \mathrm{~mm}$. This has resulted in the flooding and subsequent sagging of the biofilm, even though the position of the biofilm has not been shifted. The flooded biofilm can remain in place for over $1 \mathrm{~h}$ before finally sinking to the bottom of the microcosm. The arrow indicates the liquid level in the microcosm before the addition of KB medium. A P1000 pipette tip is visible in the background and was left in place after being used to deliver the additional KB medium. 


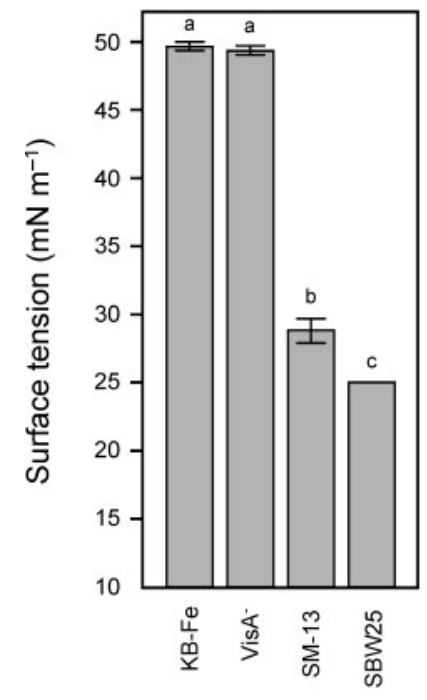

Fig. 5. Cellulose expressed by the VM biofilm contributes to a reduction in surface tension. The surface tension $(\gamma)\left(\mathrm{mN} \mathrm{m}^{-1}\right)$ of SBW25 VM biofilms was obtained by vortexing entire biofilmcontaining KB-Fe microcosms, and is compared with $\gamma$ of shaken $\mathrm{KB}-\mathrm{Fe}$ cultures of the viscosin-deficient mutant $\mathrm{Visc}^{-}$and the cellulose-deficient mutant SM-13, and sterile KB-Fe. Means were compared by Tukey-Kramer HSD $\left(\mathrm{q}_{2.861}^{*}, \alpha_{0.05}\right)$; samples not connected by the same letter are significantly different. Means \pm SEM are shown $(n=5)$.

biofilm. These investigations suggest that the weak levels of attachment and possible surface interactions can explain the localization of the VM biofilm to the A-L interface.

\section{DISCUSSION}

Although many pseudomonads are known to produce biofilms, P. fluorescens SBW25 is the first to be reported to produce submerged flow cell and static microcosm A-L biofilms, including two PC-class biofilms (WS and CBFS) and the VM-class biofilm reported here. The SBW25 VM biofilm is induced by the supplementation of $\mathrm{KB}$ static liquid microcosms with iron, as well as several other metals, and in contrast to the WS and CBFS biofilms, our results indicate that the SBW25 VM biofilm is not the result of mutation. Instead, it appears to be the result of a non-specific response to metals, including copper, iron, lead and manganese, and we have used iron as a representative metal to allow further investigation of the VM biofilm. In SBW25, it has been demonstrated that a number of different metal ions are also able to induce the transcription of a putative gene involved in copper efflux (Zhang \& Rainey, 2007); thus, such non-specificity of metal ion induction is not new. Furthermore, it has been found that iron induces cellulose expression and also contributes to the overall strength of the biofilm, most likely by enhancing matrix interconnectivity.
The mature SBW25 VM biofilm is a very fragile structure, which gives the impression of being a body of viscous liquid, rather than a discrete physical structure. However, rheological testing confirms that the VM biofilm is a viscoelastic structure rather than a viscous liquid. The physical robustness of the VM biofilm can be compared with those of both the WS and the CBFS biofilms using the direct measurements of load determined by MDM assay, which suggest that the VM biofilm is an order of magnitude weaker than these two PC-class biofilms. The shear modulus $\left(\mathrm{G}^{*}\right)$ of the VM biofilm $(0.75 \mathrm{~Pa})$ can also be compared with that of submerged flow-cell biofilms: $\mathrm{G}^{*}$ varies considerably over $0.1-1000 \mathrm{~Pa}$ (Hall-Stoodley et al., 2004) and $P$. aeruginosa biofilms have been reported with values of 1-280 Pa (Stoodley et al., 2002), indicating that the VM biofilm is one of the least robust biofilms yet investigated. Similarly, the shear stress needed to produce viscous flow in biofilms can be compared: the VM biofilm begins to flow at $0.028 \mathrm{~Pa}$, whereas in Staphylococcus aureus and $P$. aeruginosa flow-cell biofilms, this occurs at 0.26 and $950 \mathrm{~Pa}$, respectively (Körstgens et al., 2001; Rupp et al., 2005).

Although $\beta(1 \rightarrow 4)$-linked polymers such as cellulose tend to be rigid, and cross-connections often result in robust gels, some poorly connected exopolysaccharides produce viscous liquids instead (Sutherland, 2001). In the WS, cellulose is the major matrix component, but it is the interaction of the partially acetylated cellulose with attachment factor, LPS and the bacterial cells which results in a robust biofilm (Spiers \& Rainey, 2005). The fragility of the VM biofilm might therefore be explained by the lack of attachment factor, which changes the relative hydrophobicity of SBW25 cells, thereby aiding recruitment to the A-L interface, adds strength to the biofilm, and provides a means of attachment to the meniscus region of the microcosm vial.

The fragility of the VM biofilm and its globular, viscous appearance suggest that this biofilm is more similar to slimes and mucoidal colonies than the classical wellattached and robust flow-cell biofilms. Such weak structures are known to be important in plant colonization and pathogenesis, e.g. Agrobacterium colonization of root hairs, Ralstonia, Xanthomonas and Xylella infections of plant vasculature, and Erwinia and Pseudomonas syringae colonization of leaf surfaces and mesophyll tissue (Ramey et al., 2004; Danhorn \& Fuqua, 2007). These weak structures are probably maintained by internal cohesion resulting from interconnections between matrix components rather than by resistance to external forces (Klapper \& Dockery, 2006). The colonization of liquid-filled cavities and surfaces subject to low flow rates and shear might only require VM-biofilm-like physical resilience and not the enhanced robustness of flow-cell or WS and CBFS biofilms. SBW25 shows a fitness advantage when able to form a VM biofilm in $\mathrm{KB}-\mathrm{Fe}$ microcosms compared with the cellulosedeficient mutant SM-13 (A. J. Spiers \& A. Koza, unpublished observations), confirming that this fragile 
biofilm is ecologically advantageous in a static liquid microcosm [WS and CBFS mutants have a similar fitness advantage in static liquid microcosms (Spiers et al., 2002; Gehrig, 2005), whilst cellulose expression disadvantages the WS on agar plates (Spiers, 2007)].

The relatively poor levels of attachment of the SBW25 VM biofilm to the meniscus region raise the question of how this biofilm localizes to the A-L interface. In both the CBFS and WS, enhanced levels of attachment and a generally hydrophobic nature suggest that a combination of suspension and surface-tension floating (after Fidalgo et al., 2006) maintain these PC-class biofilms at the A-L interface. Although the weak levels of attachment in the VM biofilm are important, the sagging of the biofilm observed after it was flooded with additional KB suggests that surface interactions also contribute to the localization of the biofilm. The amphiphilic nature of extracellular polysaccharides (EPS) means that they can have a surfaceactive effect, and an additional role for EPS in biofilms has been posited in interface interactions (Flemming et al., 2007). Surface tension measurements indicated that the partially acetylated cellulose expressed by SBW25 in VM biofilms has a measurable surface activity, in agreement with findings for purified cellulose derivatives, which can alter the $\gamma$ of water to $37-63 \mathrm{mN} \mathrm{m}^{-1}$ (Persson et al., 1999). It is therefore possible that cellulose fibres mediate interactions between the main biofilm mass below the surface and the A-L interface itself to help maintain the biofilm at the surface.

The ability of P. fluorescens SBW25 to produce both PCand VM-class biofilms underlies the ecological advantage of being able to produce an $\mathrm{A}-\mathrm{L}$ interface biofilm, and reflects the plasticity of bacterial responses to environmental challenges. In the case of the WS and VM biofilms, cellulose is utilized to produce substantially different biofilms. In the WS, the molecular mechanism that results in cellulose and attachment factor expression and biofilm formation is reasonably well understood. Preliminary investigation of the VM biofilm has shown that the response regulator WspR involved in the WS phenotype (Spiers et al., 2002, 2003) is not required, and that transcription of the wss cellulose synthase operon is not upregulated by iron, whilst de novo transcriptiontranslation is needed, suggesting the involvement of unidentified genes in VM biofilm formation (A. J. Spiers \& A. Koza, unpublished data). Further research is also needed to determine whether SBW25 responds to increasing levels of iron (and other metals) by producing more cellulose, or whether the effect of the metal is a simple on/ off response.

\section{ACKNOWLEDGEMENTS}

We thank Marcin Skiba for photographing the biofilms. Research and A.K.'s PhD studentship were funded by the SIMBIOS Centre, University of Abertay Dundee. The University of Abertay Dundee is a charity registered in Scotland, no. SC016040. A. J.S. is a member of the Scottish Alliance for Geoscience, Environment and Society (SAGES) and A.K. is a SAGES-associated PhD student. The Scottish Crop Research Institute receives Grant-in-Aid support from the Scottish Government Rural and Environment Research and Analysis Directorate.

\section{REFERENCES}

Bantinaki, E., Kassen, R., Knight, C. G., Robinson, Z., Spiers, A. J. \& Rainey, P. B. (2007). Adaptive divergence in experimental populations of Pseudomonas fluorescens. III. Mutational origins of Wrinkly Spreader diversity. Genetics 176, 441-453.

Battin, T. J., Sloan, W. T., Kjelleberg, S., Daims, H., Head, I. M., Curtis, T. P. \& Eberl, L. (2007). Microbial landscapes: new paths to biofilm research. Nat Rev Microbiol 5, 76-81.

Branda, S. S., Vik, A., Friedman, L. \& Kolter, R. (2005). Biofilms: the matrix revisited. Trends Microbiol 13, 20-26.

Danhorn, T. \& Fuqua, C. (2007). Biofilm formation by plantassociated bacteria. Annu Rev Microbiol 61, 401-422.

de Bruijn, I., de Kock, M. J., Yang, M., de Waard, P., van Beek, T. A. \& Raaijmakers, J. M. (2007). Genome-based discovery, structure prediction and functional analysis of cyclic lipopeptide antibiotics in Pseudomonas species. Mol Microbiol 63, 417-428.

Fidalgo, M., Barrales, R. R., Ibeas, J. I. \& Jimenez, J. (2006). Adaptive evolution by mutations in the FLO11 gene. Proc Natl Acad Sci U S A 103, 11228-11233.

Flemming, H.-C., Neu, T. R. \& Wozniak, D. J. (2007). The EPS matrix: the "house of biofilm cells". J Bacteriol 189, 7945-7947.

Gehrig, S. M. (2005). Adaptation of Pseudomonas fluorescens SBW25 to the air-liquid interface: a study in evolutionary genetics. DPhil thesis, University of Oxford.

Hall-Stoodley, L., Costerton, J. W. \& Stoodley, P. (2004). Bacterial biofilms: from the natural environment to infectious diseases. Nat Rev Microbiol 2, 95-108.

Janmey, P. A. \& Schliwa, M. (2008). Rheology. Curr Biol 18, R639R641.

Janmey, P. A., Georges, P. C. \& Hvidt, S. (2007). Basic rheology for biologists. Methods Cell Biol 83, 3-27.

King, E. O., Ward, M. K. \& Raney, D. C. (1954). Two simple media for the demonstration of pyocyanin and fluorescin. J Lab Clin Med 44, 301-307.

Klapper, I. \& Dockery, J. (2006). Role of cohesion in the material description of biofilms. Phys Rev E Stat Nonlin Soft Matter Phys 74, 031902.

Körstgens, V., Flemming, H.-C., Wingender, J. \& Borchard, W. (2001). Uniaxial compression measurement device for investigation of the mechanical stability of biofilms. J Microbiol Methods 46, 9-17.

Mezger, T. G. (2006). The Rheology Handbook: for Users of Rotational and Oscillatory Rheometers, 2nd edn. Hannover, Germany: Vincentz Network.

Moon, C. D., Zhang, X.-X., Matthijs, S., Schäfer, M., Budzikiewicz, H. \& Rainey, P. B. (2008). Genomic, genetic and structural analysis of pyoverdine-mediated iron acquisition in the plant growth-promoting bacterium Pseudomonas fluorescens SBW25. BMC Microbiol 8, 7.

Persson, B., Nilsson, S. \& Bergman, R. (1999). Dynamic surface tension of dilute aqueous solutions of nonionic cellulose derivatives in relation to other macromolecular characterization parameters. J Colloid Interface Sci 218, 433-441.

Rainey, P. B. \& Bailey, M. J. (1996). Physical map of the Pseudomonas fluorescens SBW25 chromosome. Mol Microbiol 19, 521-533. 
Ramey, B. E., Koutsoudis, M., von Bodman, S. B. \& Fuqua, C. (2004). Biofilm formation in plant-microbe associations. Curr Opin Microbiol 7, 602-609.

Römling, U. (2005). Characterization of the rdar morphotype, a multicellular behaviour in Enterobacteriaceae. Cell Mol Life Sci 62, 1234-1246.

Rupp, C. J., Fux, C. A. \& Stoodley, P. (2005). Viscoelasticity of Staphylococcus aureus biofilms in response to fluid shear allows resistance to detachment and facilitates rolling migration. Appl Environ Microbiol 71, 2175-2178.

Singh, P. K., Parsek, M. R., Greenberg, E. P. \& Welsh, M. J. (2002). A component of innate immunity prevents bacterial biofilm development. Nature 417, 552-555.

Spiers, A. J. (2007). Wrinkly-Spreader fitness in the two-dimensional agar plate microcosm: maladaptation, compensation and ecological success. PLoS One 2, e740.

Spiers, A. J. \& Rainey, P. B. (2005). The Pseudomonas fluorescens SBW25 Wrinkly Spreader biofilm requires attachment factor, cellulose fibre and LPS interactions to maintain strength and integrity. Microbiology 151, 2829-2839.

Spiers, A. J., Kahn, S. G., Travisano, M., Bohannon, J. \& Rainey, P. B. (2002). Phenotypic evolution in Pseudomonas fluorescens. 1. Determinants of Wrinkly Spreader fitness and the cause of an evolutionary transition. Genetics 161, 33-46.

Spiers, A. J., Bohannon, J., Gehrig, S. \& Rainey, P. B. (2003). Biofilm formation at the air-liquid interface by the Pseudomonas fluorescens
SBW25 Wrinkly Spreader requires an acetylated form of cellulose. Mol Microbiol 50, 15-27.

Spiers, A. J., Arnold, D. L., Moon, C. D. \& Timms-Wilson, T. M. (2006). A survey of A-L biofilm formation and cellulose expression amongst soil and plant-associated Pseudomonas isolates. In Microbial Ecology of Aerial Plant Surfaces, pp. 121-132. Edited by M. J. Bailey, A. K. Lilley, T. M. Timms-Wilson \& P. T. N. Spencer-Phillips. Wallingford, UK: CABI.

Stoodley, P., Cargo, R., Rupp, C. J., Wilson, S. \& Klapper, I. (2002). Biofilm material properties as related to shear-induced deformation and detachment phenomena. J Ind Microbiol Biotechnol 29, 361-367.

Sutherland, I. W. (2001). Biofilm exopolysaccharides: a strong and sticky framework. Microbiology 147, 3-9.

Ude, S., Arnold, D. L., Moon, C. D., Timms-Wilson, T. \& Spiers, A. J. (2006). Biofilm formation and cellulose expression among diverse environmental Pseudomonas isolates. Environ Microbiol 8, 1997-2011.

Van Hamme, J. D., Singh, A. \& Ward, O. P. (2006). Physiological aspects. Part 1 in a series of papers devoted to surfactants in microbiology and biotechnology. Biotechnol Adv 24, 604-620.

Villavicencio, E. (2000). Biofilm analysis of Pseudomonas fluorescens SBW25. The role of cellulose in biofilm development. MSc thesis, Technical University of Denmark, Lyngby, Denmark.

Zhang, X. X. \& Rainey, P. B. (2007). The role of a P1-type ATPase from Pseudomonas fluorescens SBW25 in copper homeostasis and plant colonization. Mol Plant Microbe Interact 20, 581-588.

Edited by: C. Picioreanu 